\title{
IC-compatible microspectrometer using a planar imaging diffraction grating.
}

\author{
S. Grabarnik*, A. Emadi, H. Wu, G. De Graaf, G. Vdovin and R.F. Wolffenbuttel \\ Electronic Instrumentation Lab., Fac. EEMCS. Delft University of Technology, Mekelweg 4, 2628 \\ CD, Delft, The Netherlands.
}

\begin{abstract}
The design and performance of a highly miniaturized spectrometer fabricated using MEMS technologies are reported in this paper. Operation is based on an imaging diffraction grating. Minimizing fabrication complexity and assembly of the micromachined optical and electronic parts of the microspectrometer implies a planar design. It consists of two parallel glass plates, which contain all spectrograph components, including slit and diffraction grating, and can be fabricated on a single glass wafer with standard lithography. A simple analytical model for determining spectral resolution from device dimensions was developed and used for finding the optimal parameters of a miniaturized spectrometer as a compromise between size and spectral resolution. The fabricated spectrometer is very compact $\left(11 \times 1.5 \times 3 \mathrm{~mm}^{3}\right)$, which allowed mounting directly on top of an image sensor. The realized spectrometer features a $6 \mathrm{~nm}$ spectral resolution over a $100 \mathrm{~nm}$ operating range from $600 \mathrm{~nm}$ to $700 \mathrm{~nm}$, which was tested using a Ne light source.
\end{abstract}

Keywords: microspectrometer, planar diffraction grating, imaging grating

\section{INTRODUCTION}

Spectroscopic measurements are a standard tool in chemical analysis, biological and medical diagnostic and in many other industrial or scientific applications [1], [2]. Specifically, optical microspectrometers fabricated using Micro-Electro Mechanical System (MEMS) technology in silicon have a huge potential in applications where either extreme requirements on size or weight (such as space), or low costs per unit in high volume with a user-friendly interface (such as point-of-care medical diagnostics) are decisive. The benefits of a miniaturized system and the co-integration with microelectronic circuits are generally at the expense of moderate optical specifications [3]. Interference microspectrometers (mostly Fabry-Perot) and devices based on diffraction gratings have been demonstrated. Although resonator-based devices have found application in channel separation for optical communication, grating-based MEMS systems have proven more suitable for operation over a wide spectral range [3]. However, the important limitation of MEMS technology is that it is inherently planar in the sense that the out of plane dimensions can not be controlled to any value by design. Due to such a constraint the optical system of a MEMS spectrometer must contain only planar elements, which determines the use of imaging diffraction optics. A flat imaging diffraction grating combines collimating, dispersion and focusing in a single optical element thus simplifying the spectrometer design considerably. Imaging gratings are relatively easy to implement in waveguide-based systems and compact spectrometer have been described in the literature [4] - [8]. Classical spectrometers with a planar diffraction grating have also been demonstrated [9]. These microspectrometers have a volume typically in the $1 \mathrm{~cm} 3$ range with a $5 \mathrm{~nm}$ resolution within $100 \mathrm{~nm}$ spectral range. A promising result was obtained employing integrated optics for the realization of a spectrometer with the largest dimension of about $1.2 \mathrm{~cm}$ and a spectral resolution varying from $0.3 \mathrm{~nm}$ to $4.6 \mathrm{~nm}$ in the $150 \mathrm{~nm}$ wavelength range [7]. In [8] the authors expect for a waveguide spectrometer a theoretically feasible resolution of $2 \mathrm{~nm}$ within a $300 \mathrm{~nm}$ operating bandwidth based on numerical simulations. Several planar diffraction gratings can be combined to improve the imaging properties of an optical system [10]. Such an approach applied to a spectrometer can result in an increased spectral resolution [11], [12].

The objective of this work is to design and fabricate a microspectrometer with the best possible spectral resolution under the dimensional and technological constraints and with a volume at least an order of magnitude smaller than the current state of the art The optimal design parameters were found using an analytical model of a spectrometer based on a single imaging diffraction grating.

*s.grabarnik@tudelft.nl; phone 31 15 278-6285; fax 31 15 278-5755

Micro-Optics 2008, edited by Hugo Thienpont, Peter Van Daele, Jürgen Mohr, Mohammad R. Taghizadeh, Proc. of SPIE Vol. 6992, 699215, (2008) · 0277-786X/08/\$18 · doi: 10.1117/12.781103 
An analysis of the imaging properties of a single-wavelength optical system employing diffraction optical elements has been presented, for example, in the articles [10] and [13]. In this article, such an analysis was combined with the diffraction grating theory [14], [15] and applied to the problem of the design of a microspectrometer. As a result an analytical model of a spectrometer was developed. It takes into account such factors as optical aberrations and diffraction effects, which do significantly influence the spectral resolution of a spectrometer when scaling down the characteristic dimension to the millimeter range. The model covers a number of generic spectrometer designs based on a planar imaging diffraction grating and allows the estimation of the best achievable spectral resolution of a spectrometer at its main design parameters, minimizing the dimensions of the device.

The fabrication concept builds on the understanding that the optical performance critically depends on the alignment of the components in the microspectrometer system. As a consequence, the planar spectrometer design is composed of two parallel glass plates and mounted directly on the surface of an imaging sensor. All spectrograph components, including the input slit and the diffraction grating, are fabricated on one plane wafer. Thus the slit and the grating are automatically aligned. Fabrication starts with a single glass wafer and IC-compatible deposition and lithography are applied to realize the parts in aluminum. The design allows relaxed alignment tolerances of the two glass plates after dicing: $15 \mu \mathrm{m}$ for the spacing and $100 \mu \mathrm{m}$ lateral misalignment of the glass plates, which highly simplifies assembly of the device. The fabricated device was mounted directly on top of an image sensor, takes a volume of only $1.5 \times 3 \times 11=50 \mathrm{~mm}^{3}$ and features a $6 \mathrm{~nm}$ spectral resolution in a $100 \mathrm{~nm}$ operating range from $600 \mathrm{~nm}$ to $700 \mathrm{~nm}$.

\section{THEORETICAL BASIS OF THE SPECTROMETER MODEL.}

The resolution of a spectrometer can be calculated as a product of the diffraction grating dispersion and the spot size produced by the imaging system when the entrance slit is illuminated with a monochromatic light. Following the analysis of a single-wavelength imaging system in [10] and [13], the spot size can be estimated as a sum of different contributions including diffraction broadening and aberrations. In case of a spectrometer the width of the image of the entrance slit should also be considered. The calculation of the dispersion, the diffraction broadening and the entrance slit image is straightforward. In order to take the aberrations into account the geometrical theory of the diffraction gratings [14], [15] can be used. Figure 1(a) presents the geometry of a diffraction grating which images point $A$ into point $B$. Plane $y z$ of the coordinate system oxyz lies in the grating plane and the origin of the coordinate system, $o$, coincides with the grating central point $O$. Consider point $O$ and a point $P(y, z)$ somewhere in the grating plain. Then the optical path difference between light beams passing from $A$ to $B$ via point $P$ and via point $O$ is:

$$
F(\lambda, y, z)=A P B-A O B+m \lambda N(y, z),
$$

where $N(y, z)$ is the number of grooves between points $O$ and $P(y, z), m$ is a diffraction order and $\lambda$ is a wavelength. The function in Eq. (1) can be decomposed into power series and take the form:

$$
F(\lambda, y, z)=\sum_{i=0}^{\infty} \sum_{j=0}^{\infty} F_{i j} y^{i} z^{j}=\sum_{i=0}^{\infty} \sum_{j=0}^{\infty}\left(M_{i j}+m \lambda N_{i j}\right) y^{i} z^{j}
$$

A path difference $F$ in Eq. (2) equal to zero corresponds to the aberration-free convergent wavefront. Thus, the coefficients $F_{i j}$ define deviations of the wavefront proportional to $y^{i} z^{j}$ and can be considered as the aberration coefficients. The coefficients $M_{i j}$ depend on the system configuration and the coefficients $N_{i j}$ are determined by the grating groove pattern [15]. For the wavelength $\lambda_{0}$ the coefficients $N_{i j}$ can be selected in such a way that the aberration coefficients $F_{i j}$ are zero, thus yielding an aberration-minimum image of a point $A$. Given the geometrical parameters of the system, the grating coefficients can be calculated analytically:

$$
N_{i j}=-\frac{M_{i j}}{m \lambda_{0}}=\frac{1}{i ! j ! m \lambda_{0}}\left[\frac{\partial^{i+j}(A P B-A O B)}{\partial y^{i} \partial z^{j}}\right]_{(0,0)}
$$

Exact formulae for $N_{i j}$ are listed for example in [15]. The groove pattern defined by the coefficients $N_{i j}$ resembles a part of a diffraction lens, since it takes the shape of a set of curved grooves not equally distributed in the grating plane. The local period of such a grating along the oy axis is $d=(\partial N / \partial y)^{-1}$. The period $d$ at the central grating point is referred to as the grating period $d_{0}$. The parameter $\lambda_{0}$ in Eq. (3) is the design wavelength, which is the central wavelength of the spectrometer operating bandwidth. 

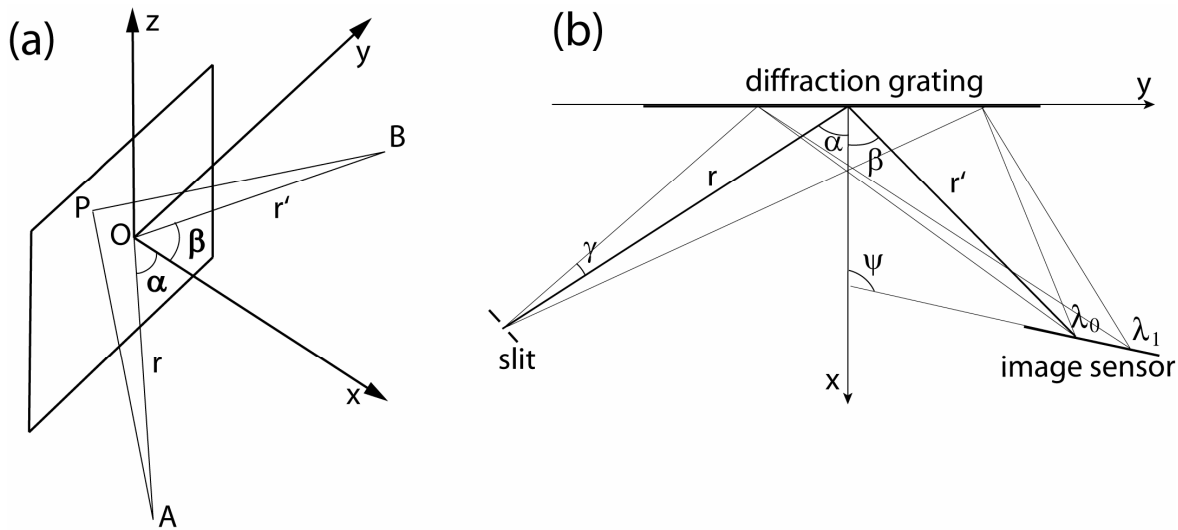

Fig. 1. Diffraction grating imaging point A into point B (a) and a general spectrometer layout (b).

A schematic of a spectrograph setup is shown in Fig. 1(b). In the design optimization only the xoy plane is considered, since this is the dispersion plane of the grating. Incident light entering through the entrance slit is directed to the grating at the angle $\alpha$ with the grating normal and diffracts towards the photodetector array at the angle $\beta$ relative to the normal. The parameters of the design are: the incident angle $\alpha$, input aperture angle $\gamma$ defining the numerical aperture $N A=\sin (\gamma)$, distance $r$ from the input slit to the grating surface, grating period $d_{0}$, position of the image sensor plane (determined by the distance $r$ ' and angle $\psi$ ) and the design wavelength $\lambda_{0}$. The size of the illuminated part of the grating, $L=y 1-y 2$, is defined by $r, \alpha$ and $\gamma$ and can be calculated from the simple geometrical considerations, as shown in Fig. 1(b). At any set of design parameters the resolution of such a spectrometer can be determined considering the contributions of the relevant factors: optical aberrations, diffraction broadening of the image, influence of the slit width.

An imaging diffraction grating designed using Eq. (3) to calculate the grating coefficients reduces aberrations at the design wavelength $\lambda_{0}$. However, for the wavelengths not equal to $\lambda_{0}$, the optical path difference (Eq. (2)) is no longer zero and the image of the slit in the detector plane is broadened. This angular broadening can be calculated from the geometrical considerations using the equation for transverse ray aberrations [16]:

$$
s_{\text {aberr }}=\left.\frac{\partial F}{\partial y}\right|_{y=y 1}+\left.\frac{\partial F}{\partial y}\right|_{y=y 2}
$$

For a finite number of the row members in Eq. (2) the value of $s_{\text {aberr }}$ can be calculated analytically using Eqs. (2), (3) and (4). Note that the coefficients $N_{i j}$ in the Eq. (2) are calculated only once for the design wavelength, while the coefficients $M_{i j}$ should be recalculated for another value of the wavelength taking into account that values of $\beta$ and $r$ ' change with the wavelength.

The spatially finite size of the illuminated part of the grating leads to the diffraction broadening of the image of a point source. Such a broadening can be estimated as follows:

$$
S_{\text {diffr }}=\frac{2 \lambda}{y 2-y 1}
$$

The width of the entrance slit also influences the spectral resolution since the smaller the entrance slit the smaller its image on a photodetector. However there is an optimal slit width defined by the input aperture and reducing the entrance slit further would not improve the resolution. The angular width of the entrance slit image is:

$$
S_{\text {slit }}=\frac{\cos (\alpha)}{\cos (\beta)} \times \frac{s_{0}}{r}
$$

where $s_{0}=\lambda / \gamma$ is the optimal width of the entrance slit. 
The resolution of the spectrometer $\Delta \lambda$ can be found as the product of the angular dispersion and the sum of different contributions given by the Eqs. (4) - (6):

$$
\Delta \lambda=\Delta \lambda\left(\lambda, \lambda_{0}, \alpha, r, \beta, r^{\prime}, \gamma, \psi\right)=f \times\left(s_{\text {slit }}+s_{\text {aberr }}+s_{\text {diffr }}\right)
$$

The angular dispersion of the grating is defined by:

$$
f=d_{0} \times \cos (\beta)
$$

Equations (1) - (8) are the basis of the model describing the dependence of the spectrometer resolution on different parameters. It should be emphasized that these equations (except for Eq. 5) result from a geometrical optics approach and provide an estimate of the resolution based on the calculation of the entrance slit image width, multiplied by the dispersion. However, geometrical optics cannot provide precise values of the resolution, since a diffraction analysis should be used that takes into account the phase and interference of the light. An accurate resolution estimate can be obtained via calculation of the point spread function (PSF) of the imaging system, convoluted with the transmission function of the entrance slit. The width of this curve measured at half maximum level (FWHM) and multiplied by the dispersion gives an accurate value for the resolution. For a spectrometer with optical aberrations that are small compared to the diffraction-limited performance of the system, the estimation of resolution based on the geometrical optics would give a better value than the diffraction analysis. Equation (5) was included in the model to compensate for such a discrepancy. In case of a system with relatively large optical aberrations, which is typically the case in a microspectrometer, the geometrical approach would provide an underestimated result. In this case the Eqs. (1) - (8) provide an estimate of the resolution defined as a full width of a monochromatic spectral line at zero level, which implies that the resolution of an actual spectrometer defined as the width of the monochromatic line at half maximum (FWHM) is approximately two times better than one predicted by the model. Therefore, this factor of 2 is included in the resolution specifications in terms of FWHM that result from the analytical modeling discussed in this paper.

\section{ANALYSIS OF THE MODEL}

According to Eq. (7), the resolution of a spectrometer depends on 8 parameters: $\lambda, \lambda_{0}, \alpha, r, \beta, r^{\prime}, \gamma$ and $\psi$. The number of the independent parameters can be reduced when considering a specific design problem. Usually the operating spectral range is fixed, so $\lambda$ and $\lambda_{0}$ can be set to fixed values. Then, we can define a grating period $d_{0}$ thus removing the diffraction angle $\beta$ from the list of parameters. Generally, the value of $d_{0}$ is defined by the technology constraint and for a lithographically fabricated grating such a constraint is the lithography resolution. Finally, the values of $r$ and $r$ ' can be set equal to the dimensional constraints and angle $\psi$ can be optimized to find the best resolution for two independent parameters $\alpha$ and $\gamma$. For example, Fig. 2 shows the dependence of the resolution on the incident angle $\alpha$ and the input aperture angle $\gamma$ within the operating bandwidth of $100 \mathrm{~nm}$.

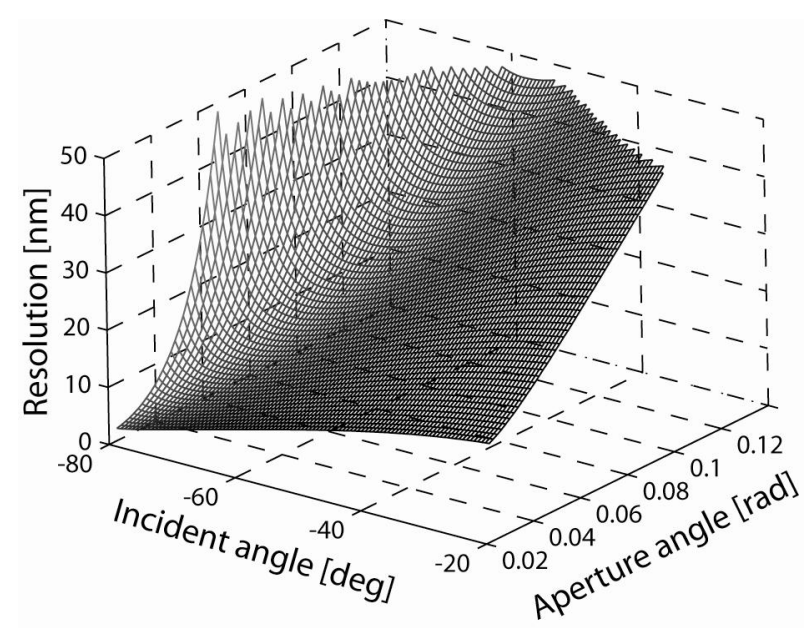

Fig. 2. Diffraction grating imaging point A into point B (a) and a general spectrometer layout (b). 
The fixed parameters in Eq. (7) are due to design targets and are set at: $r=r^{\prime}=10 \mathrm{~mm}$ and $\lambda_{0}=650 \mathrm{~nm}$ and those due to technological constraints at: $\mathrm{d}_{0}=2 \mu \mathrm{m}$ and $\psi=\pi / 2$. From Fig. 2 it is evident that a small aperture angle and a large angle of incidence contribute to an improved resolution of the spectrometer. The improved spectral resolution with reduced aperture complies with intuition, since aberrations of the grating are the main contribution to the resolution function in Eq. (7). However, the input aperture angle also defines the optical throughput (or luminosity) of the spectrometer. The resulting signal reduction at the photodetector reduces the overall sensitivity of the device.

Since spectral resolution and throughput are not independent parameters it is necessary to fix the spectrometer input aperture to such a value that an acceptable signal-to-noise ratio results at the detector and to take the angle $\psi$ as a variable. Also at this set of fixed parameters the dependence of the resolution within a specified wavelength range on the other design parameters such as the incidence angle and distance from the slit to the grating $r$ can be investigated. Assume further that $r^{\prime}=r$. Since $r$ is the largest dimensional parameter in the design it is convenient to take it as a measure of the spectrometer size and in the following discussion $r$ is referred to as the spectrometer size or dimension. The angle $\psi$ between photodetector plane and the grating normal can be optimized to maximize resolution. It should be noted that the optimal value of the angle $\psi$ does not depend on the spectrometer size. This statement is supported when considering the characteristics of a diffraction lens. The focal distance of such a lens is inversely proportional to wavelength. That is why the optimal value of $\psi$ is defined by the angle of incidence and grating period. Since the angle at which light falls on the surface of a photodetector should not exceed some maximum value to avoid Fresnel reflection of a substantial part of radiation, this angle is limited in the model to 45 degrees. Using Fig. 1 yields: $\psi_{\max }=180-45-$ $\beta$.
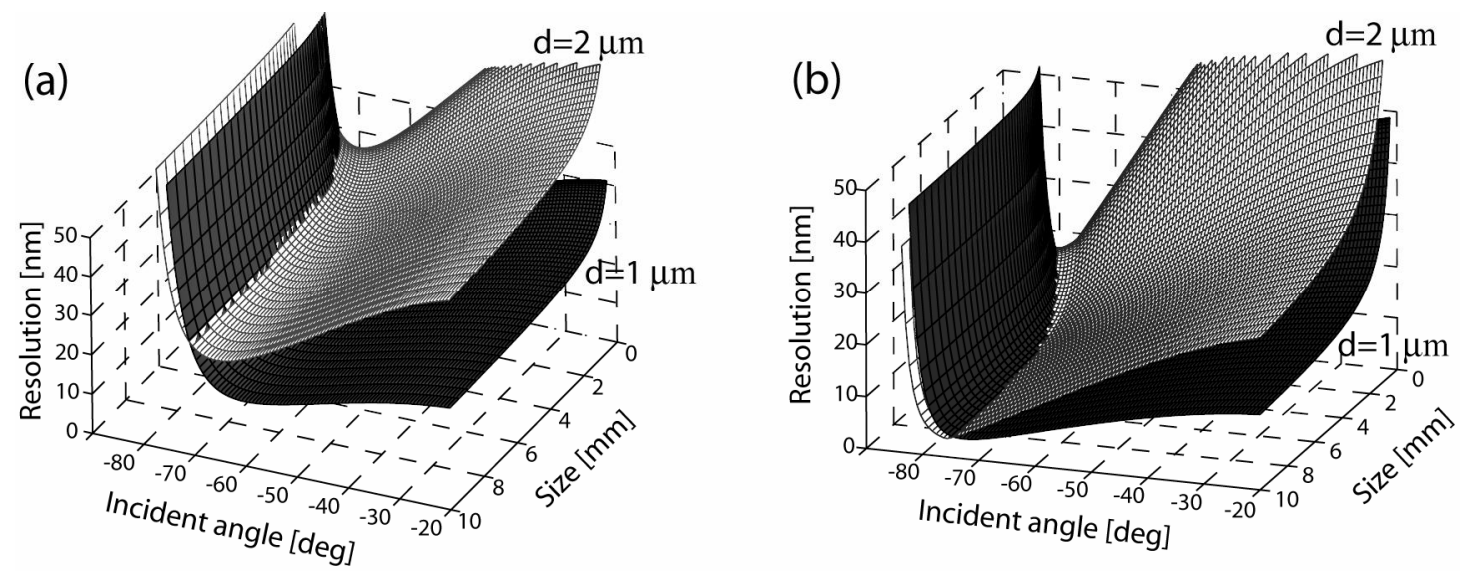

Fig. 3. The dependence of spectral resolution on the incident angle and the distance from the grating to the entrance slit $r$ (the size of the device). The results are for the input aperture angle of $0.1 \mathrm{rad}$. (a) and $0.05 \mathrm{rad}$. (b) are presented.

Figure 3 shows the spectrometer resolution depending on the angle of incidence and on spectrometer dimension. These dependencies were calculated for the operating range of $100 \mathrm{~nm}$ and two different values of the grating period. Results for the aperture angle of $0.1 \mathrm{rad}$. are presented in Fig. 3(a) and results for an aperture of 0.05 rad. are shown in Fig. 3(b). For each data point on the Fig. 3 the value of $\psi$ was optimized. The optimal value gradually varies depending on the incidence angle from $\sim 90^{\circ}$ corresponding to the incidence angle of $20^{\circ}$ to $\sim 130^{\circ}$ for incidence angle of $80^{\circ}$. As can be derived from Fig. 3, the resolution is weakly depending on the size of the spectrometer in case the size is larger then 5 $4 \mathrm{~mm}$. The best results are achieved at an angle of incidence in the $70^{\circ}$ to $75^{\circ}$ range, irrespective of spectrometer size. The strong dependence of the resolution on angle of incidence is due to aberrations of the grating, which is the main contribution in the resolution dependence. The width of the entrance slit image and the diffraction broadening start to affect resolution for a spectrometer dimension smaller than $2-3 \mathrm{~mm}$. This dependence is inversely proportional to $r$, which is in agreement with Eq. (6). For the optimal width of the entrance slit, the influence of the slit image and the influence of the diffraction broadening on the resolution are of the same order of magnitude. Figure 3 predicts a resolution of a plane grating spectrometer with dimension as small as $6 \mathrm{~mm}$ of about $5-10 \mathrm{~nm}$ within a $100 \mathrm{~nm}$ operating range provided that the numerical aperture of the spectrometer is sufficiently small ( about 0.05 ). This result is the starting point in the microspectrometer design presented below. 


\section{DESIGN OF A MICROSPECTROMETER}

The simplified structure of the planar imaging grating based microspectrometer is shown in Fig 4. The spectrometer consists of two pieces of glass aligned parallel to each other. All spectrometer optical components, including the input slit and the diffraction grating operate in reflection. The light from a source is reflected from a stripe mirror, acting as a slit and then redirected by the upper mirror 1 to the diffraction grating. The diffracted light is reflected by the mirror 2 and finally projected onto the photodetector (CCD imager) through the glass plate. Hence, the imaging system of the spectrometer projects the dispersed image of the reflective input slit onto the plane of the image sensor. In this sense the operation of the reflective slit is similar to the conventional slit in a classical spectrometer. The width of the slit mirror is $50 \mu \mathrm{m}$, which corresponds to the effective slit width of $25 \mu \mathrm{m}$ when taking into account the $60^{\circ}$ angle of incidence. The dimensions of mirror 1 define the numerical aperture of the spectrometer, since the non-reflected light escapes the system through the glass plate. As was already discussed in Section 3, the value of the angle of incidence in the range of 70 - 75 degrees would give a superior performance (Fig. 2), however the amount of light reflected from the glass surfaces increases with incident angle, due to Fresnel reflection. Such parasitic reflections contribute to the stray light, which is indeed observed in the fabricated device and is discussed in more detail in the next section. Therefore, the design was based on an angle of incidence at 60 degrees, as a compromise between stray light level and resolution.

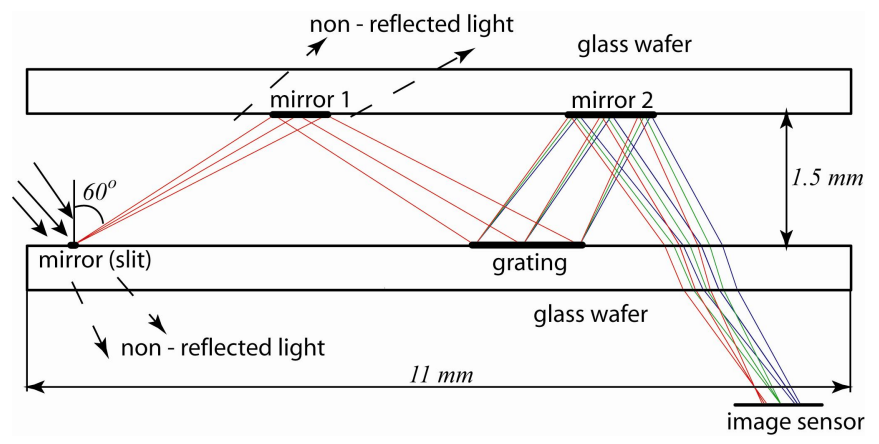

Fig. 4. Design of the spectrometer.

The period of the grating $d_{0}$ (the local period in the grating center) should be minimized to achieve best resolution, as results from Fig. 3. For the lithographically fabricated grating the smallest period is constrained by the resolution of the available lithographic technology and is two times larger than the minimum feature size of the lithographic process. Taking into account that the local period varies along the grating and that the resolution of the available lithography was $0.7 \mu \mathrm{m}$, the parameter $d_{0}$ was chosen to be $2 \mu \mathrm{m}$. The numerical aperture was selected at 0.05 and the distance between the glass plates was $1.5 \mathrm{~mm}$, which yields the very compact device shown in Fig. 4. The angle $\psi=90^{\circ}$ (see Fig. 1) is determined by the direct mounting of the image sensor in a planar structure. The optimal value according to the model, $\psi$ $=102^{\circ}$, results in the improvement of the resolution by about $12 \%$, as compared to the set-up with $\psi=90^{\circ}(13 \mathrm{~nm}$ versus $14.6 \mathrm{~nm}$, which is equivalent to $6.5 \mathrm{~nm}$ FWHM and $7.3 \mathrm{~nm}$ FWHM respectively). The spectrometer design has been verified using rigorous ray tracing software ZEMAX [17]. The results of the model were in a good agreement with the resolution estimate based on geometrical ray tracing using ZEMAX. However accurate calculation based on the PSF in ZEMAX resulted in a resolution of $5.6 \mathrm{~nm}$ FWHM. Hence, the analytical design provides a $30 \%$ underestimated result. In the final design step ZEMAX was used to estimate tolerances for the alignment of two spectrometer parts. The influence of the lateral misalignment and the errors in spacing between two glass plates on the spectral resolution were analyzed using ZEMAX PSF cross section calculation. Any displacement of the glass plates results in a mirrors (mirrors 1 and 2 in Fig. 4) displacement and thus in a change of the angle of incidence and the length of optical pass that light travels from the slit to the grating. Such an error is the reason for the aberrations of the grating not to be minimized at the design wavelength, but rather at a wavelength spectrally shifted from the designed value. Thus, a misalignment within reasonable bounds results in a small shift of the operating bandwidth. Having limited such a shift to $10 \mathrm{~nm}$ we obtained that the alignment tolerances are approximately $15 \mu \mathrm{m}$ for the spacing and $100 \mu \mathrm{m}$ for the lateral alignment of the glass plates. According to ZEMAX PSF calculations the resolution is decreased by less than $10 \%$ within the shifted operating bandwidth.

Having the necessary design parameters defined, Eq. (3) can be used to calculate the grating coefficients. Subsequently, for each of the grating grooves a set of points $(y, z)$ that are part of the groove and describe its position in the grating plane was determined. This procedure enabled generating the mask using IC design software tools. The grating consisted 
of curved grooves. The line spacing varies from $1.5 \mu \mathrm{m}$ to $2.8 \mu \mathrm{m}$ along the grating. Since the lithographic technique did not allow fabrication of blazed gratings, the only physical parameter available for optimization was the depth of the rectangular grating grooves, which was chosen to be equal to $230 \mathrm{~nm}$ maximizing the diffraction efficiency for an Alcoated grating in the wavelength region from $600 \mathrm{~nm}$ to $700 \mathrm{~nm}$. The resulting diffraction efficiency equals $38 \%$ at wavelength $630 \mathrm{~nm}$ and $29 \%$ at wavelength $680 \mathrm{~nm}$. These values are low compared to the blazed gratings efficiency with the peak in the range of $70 \%-80 \%$. A more-complicated fabrication technology is required for increasing the efficiency of the grating used in a microspectrometer.

\section{FABRICATION AND CHARACTERIZATION OF THE SPECTROMETER}

All elements of the spectrometer, including the slit and the gratings, were fabricated on a single glass wafer with a 2mask lithography process. First, $650 \mathrm{~nm}$ of aluminum was deposited and the first mask was applied to define the grating structures. Subsequently the gratings were plasma etched to yield $230 \mathrm{~nm}$ deep grooves in the aluminum. Finally, using the second mask, the aluminum was patterned and etched to define the input slit, the grating and the mirrors. The grooves, which were defined in the first step, were protected during the etching. Figure 5 presents SEM (Scanning Electron Microscope) photographs of the grating.
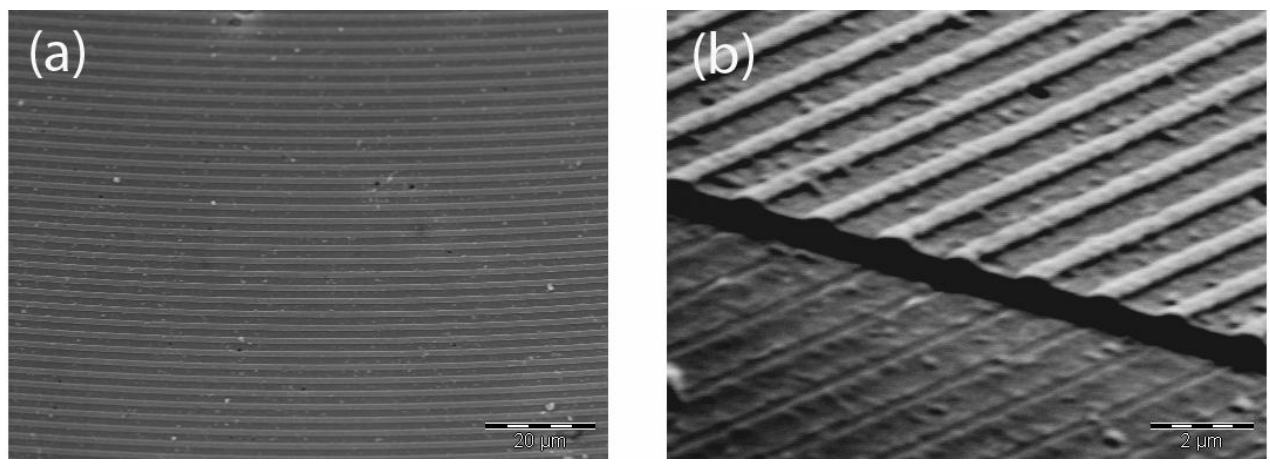

Fig. 5. SEM photograph of the central part of the grating (a) and the grating edge (b).

The curvature of the grooves is clearly visible in Fig. 5(a). Figure 5(b) reveals that the profile of the grooves is not rectangular. This is due to the rather narrow width of the grooves. The width was at the limit of the lithography resolution and thus the images of the lines on the photoresist that determined the pattern to be etched were blurred. This effect resulted in the rounding of the grooves edges during the etching process. Such rounded edges do not influence the imaging properties of the grating, however the diffraction efficiency can be reduced Measurements of the grating efficiency were performed at a wavelength of $632 \mathrm{~nm}$ using a He-Ne laser and demonstrated an efficiency of $35 \%$ in the first diffraction order for the incident angle of 60 degrees.

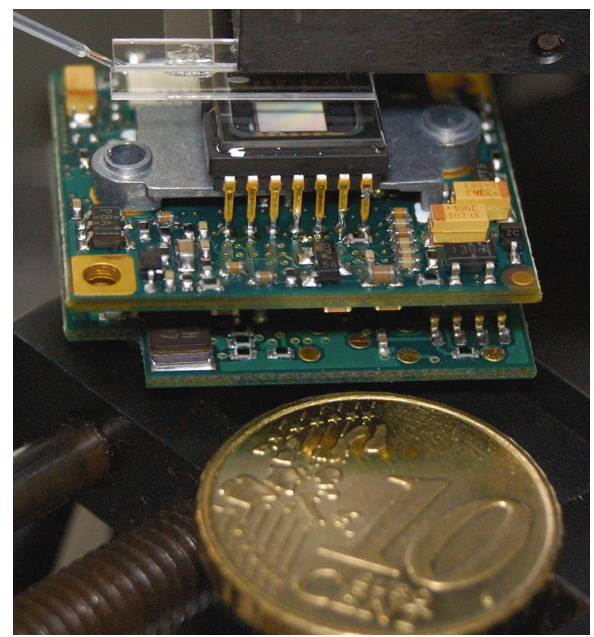

Fig. 6. Planar microspectrometer. Light is deliverd to the device using an optical fiber visible in the left top corner. 
The processed wafer was diced and the two parts of the spectrometer were aligned and mounted on top of the CCD imaging sensor, as shown in Fig. 6. A multi-mode optical fiber was used to feed the optical signal into the microspectrometer. One of the cleaved fiber tips was exposed to a light source under test and the second fiber tip illuminated the input slit of the spectrometer. As an example of the device performance, Fig. 7 represents the spectrum of a Ne lamp captured with the device in the range between $630 \mathrm{~nm}$ and $730 \mathrm{~nm}$.

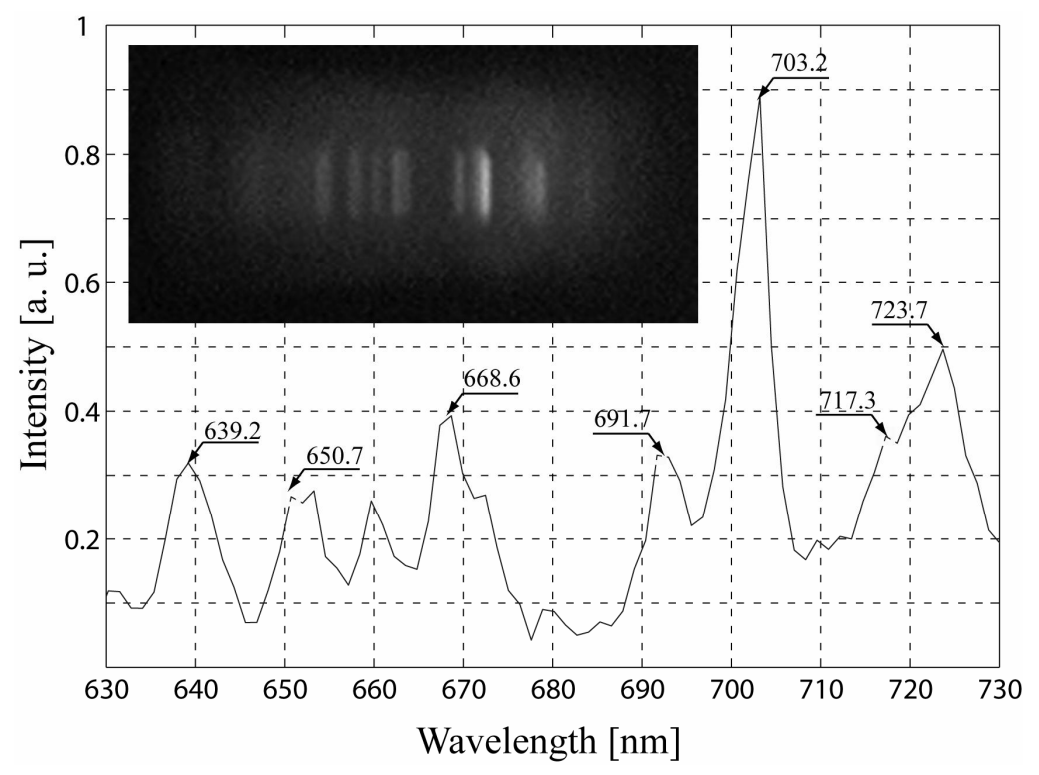

Fig. 7. Ne spectrum captured with the CCD camera and the graphical representation of this spectrum.

The setup presented on Fig. 6 was equipped with several microscrews, which allow the adjustment of the position of the upper glass plate, while the position of lower plate was fixed just above the image sensor. The optimal position of the upper plate was found experimentally by adjusting until the best spectral pattern with sharp lines was obtained. Moving the top glass plate up, down, left and right from this optimal position within the tolerance bounds defined at the design stage did not result in an observable degradation of spectral resolution.

The effect of stray light is clearly visible in Fig 7. The Fresnel reflection of the glass surface around the input slit is, at the given angle of incidence $\left(60^{\circ}\right)$, about $9 \%$ of the light impinging on the input slit (see Fig. 4), whereas reflectance of aluminum is about $90 \%$ in the visible spectral range [18]. Hence, the contrast is just a factor 10 . The spectral image presented in the insert in Fig. 7 is actually a superposition of the dispersed images of the entrance slit and the light spot of the fiber tip that was used for illumination of the slit. The elimination of Fresnel reflections would substantially decrease the level of stray light. It could be achieved by depositing an appropriate antireflection coating on the unused surfaces. The coating process would involve an additional lithography step: the deposition of an appropriate material on top of a wafer followed by the patterning of this material to open aluminum gratings and mirrors and that part of the glass plate which covers the image sensor. Implementation of such a coating is part of our future work.

The major spectral lines are marked in Fig. 7 to identify them with the Ne spectral lines. From Fig. 7 we estimate the FWHM resolution to be approximately $6 \mathrm{~nm}$, while the relative positions of spectral lines can be determined with a precision of better than $1 \mathrm{~nm}$. Hence, the measured resolution of the spectrometer is $20 \%$ better than the analytical predictions based on geometrical approach presented in Fig.3(b) and is in a good agreement with the estimates obtained using the ZEMAX PSF calculation.

\section{CONCLUSIONS}

The spectrometer design process started with the generalization of the design problem on the basis of an analytical model. The simulations not only confirmed the reasonable expectation that optical aberrations are the major limiting factor to the spectral resolution of a miniaturized spectrometer, but also facilitated the design of the actual microspectrometer with dimensional constraints. The analysis of the model demonstrated that the best resolution of the 
spectrometer could be achieved with high incident angles (about $70^{\circ}-75^{\circ}$ ) and small input aperture angles(about 0.05 rad.). Practical constraints led to a configuration that slightly departs from the model optimum. Fresnel reflections limited the incident angle to 60 degrees and due to the design planarity the image sensor was parallel to the grating plane. With such constraints the FWHM resolution of the designed spectrometer was predicted to be about $5.6 \mathrm{~nm}$. The planar design simplifies its fabrication and assembly considerably. The highly miniaturized (dimensions only $11 \times 1.5 \times 3 \mathrm{~mm}^{3}$ ) spectrometer demonstrated a FWHM spectral resolution of $6 \mathrm{~nm}$ within a $100 \mathrm{~nm}$ operating range, which is in good agreement with the simulation results. The measured resolution of the spectrometer is $20 \%$ better than the analytical predictions based on the geometrical approach presented in Fig.3 and is in a good agreement with the estimates obtained using a ZEMAX PSF calculation. Although the PSF approach has been demonstrated to yield a more accurate result, the analytical approach based on geometrical optics presented here is more suitable for the interactive design of a microspectrometer with strict dimensional constraints.

Improvement of the resolution is possible if the design or technology constraints are relaxed. The most important constraint is the one for the incident angle resulted from the Fresnel reflection, as was discussed in Sections 4 and 5. If such reflections are eliminated using an antireflection coating the incident angle can be increased to the optimal value of about 76 degrees, resulting in an improvement in spectral resolution by a factor 3, which implies that a FWHM resolution below $2 \mathrm{~nm}$ is possible. A better spectrometer performance can also be achieved with a larger grating groove density. This would be possible with a higher resolution lithography process. The relaxed dimensional constraint cannot improve the resolution considerably as shown in Fig. 3. For example, increasing the size of the device from $6 \mathrm{~mm}$ to 10 $\mathrm{mm}$ results in an improvement of the resolution of less than $10 \%$. The last constraint is the numerical aperture, which is already small. However, for applications involving bright sources or sources with limited aperture this constraint is not an issue.

Thus, future work will be directed towards the improvement of the planar spectrometer with respect to the elimination of Fresnel reflections at unused surfaces of the device and on the IC-compatible fabrication of more-efficient diffraction gratings.

\section{ACKNOWLEDGEMENT}

The authors acknowledge the financial support from the Dutch Technical Foundation (STW), grant DET.6667. We are indebted to Lina Sarro and Charles de Boer (DIMES, TU Delft) for the very useful discussions on fabrication technology and wafer processing and to Jan Groeneweg and Jan Cornelis Wolff (DIMES, TU Delft) for the mask fabrication.

\section{REFERENCES}

1 R. J. Dempsey, D. G. Davis, R. G. Buice, Jr., and R. A. Lodder, "Biological and medical applications of nearinfrared spectroscopy," Appl. Spectrosc. 50, 18A-34A (1996).

2 C. P. Bacon, Y. Mattley, and R. DeFrece, "Miniature spectroscopic instrumentation: application to biology and chemistry," Rev. Sci. Instrum. 75, 1-16 (2004).

3 R.F. Wolffenbuttel, "MEMS-based optical mini- and microspectrometers for the visible and infrared spectral range", J. Micromech. Microeng. 15, S145-S152, (2005).

4 H. W. Yen, H. R. Friedrich, R. J. Morrison and G. L. Tangonan, "Planar Rowland spectrometer for fiber-optic wavelength demultiplexing," Opt. Lett. 6, 639-641 (1981).

5 D.S. Goldman, P.L. White and N.C. Anheier, "Miniaturized spectrometer employing planar waveguides and grating couplers for chemical analysis," Appl. Opt. 29, 4583-4589 (1990).

6 D. Sander and J. Muller, "Selffocusing phase transmission grating for an integrated optical microspectrometer," Sens. Act. A 88, 1-9 (2001).

7 K. Chaganti, I. Salakhutdinov, I. Avrutsky and G. W. Auner, "A simple miniature optical spectrometer with a planar waveguide grating coupler in combination with a plano-convex lens," Opt. Express 14, 4064-4072 (2006).

8 I. Avrutsky, K. Chaganti, I. Salakhutdinov and G. Auner, "Concept of a miniature optical spectrometer using integrated optical and micro-optical components," Appl. Opt. 45, 7811-7817 (2006).

9 S. Ura, F. Okayama, K. Shiroshita, K. Nishio, T. Sasaki, H. Nishihara, T. Yotsuya, M. Okano and K. Satoh, "Planar Reflection Grating Lens for Compact Spectroscopic Imaging System,” Appl. Opt. 42, 175-180 (2003). 
10 M. Testorf and J. Jahns, "Imaging properties of planar-integrated micro-optics”, JOSA A 16, 1175-1183 (1999).

11 S. Ura, T. Sasaki and H. Nishihara, "Combination of Grating Lenses for Color Splitting and Imaging ," Appl. Opt. 40, 5819-5824 (2001).

12 S. Grabarnik, R. Wolffenbuttel, A. Emadi, M. Loktev, E. Sokolova, and G. Vdovin, "Planar double-grating microspectrometer," Opt. Express 15, 3581-3588 (2007).

13 R. K. Kostuk, J. W. Goodman and L. Hesselink, "Design considerations for holographic optical interconnects," Appl. Opt. 26, 3947-3953 (1987).

14 H. Noda, T. Namioka and M. Seya, "Geometric theory of the grating," J. Opt. Soc. Am. 64, 1031-1036 (1974).

15 C. Palmer and W.R. McKinney, "Imaging theory of plane-symmetric varied line-space grating systems", Opt. Eng., 33, 820-829 (1994).

16 M. Born and E. Wolf, Principles of Optics (Cambridge Univ. Press, Cambridge, 2002)

17 ZEMAX Optical Design Program, User's Guide, Version 9.0 (Focus Software, Inc., Tucson, Ariz., 2000).

18 D. Rossberg, Optical properties of the integrated infrared sensor, Sensors and Actuators A 54, 793-797 (1996). 